\title{
QUANTITATIVA OU QUALITATIVA? UM ALINHAMENTO ENTRE PESQUISA, PESQUISADOR E ACHADOS EM PESQUISAS SOCIAIS
}

\section{QUANTITATIVE OR QUALITATIVE? ALIGNMENT BETWEEN RESEARCH, RESEARCHER AND FINDINGS IN SOCIAL RESEARCH}

\begin{abstract}
Luciano Ferreira da Silva
Docente e pesquisador no Mestrado Profissional em Administração - Gestão de Projetos na Universidade Nove de Julho - UNINOVE São Paulo, SP, Brasil

Email: If_silvabr@yahoo.com.br
\end{abstract}

Rosária de Fátima Segger Macri Russo

Docente e pesquisadora no Mestrado Profissional em Administração - Gestão de Projetos na Universidade Nove de Julho - UNINOVE São Paulo, SP, Brasil

Email: romacrirusso@gmail.com

Paulo Sergio Gonçalves de Oliveira

Docente e pesquisador no Mestrado Profissional em Gestão de Alimentos e Bebidas da Universidade Anhembi Morumbi

São Paulo, SP, Brasil

Email: psgoliveira@hotmail.com

\section{RESUMO}

O presente artigo teórico conceitual teve como objetivo refletir a respeito de uma relação coerente entre paradigma, métodos e achados de uma pesquisa. Esse artigo se propõe a fomentar o debate com pesquisadores, além de editores e revisores de revistas acadêmicas. A discussão aqui apresentada segue de forma heurística um debate com base em obras que tratam principalmente sobre pesquisas na área das ciências sociais tendo como foco a área de administração. O problema aqui evidenciado é o de o pesquisador adotar primeiramente como posicionamento de sua pesquisa uma abordagem metodológica. Destaca-se que o erro está em defender pura e simplesmente uma estratégia metodológica como se essa fosse o paradigma de pesquisa. Assim, um alinhamento natural seria estabelecer uma relação do paradigma de pesquisa para uma problemática, e dessa para uma estratégia metodológica. Espera-se com a discussão aqui apresentada contribuir com o conhecimento que será construído com base em uma tríplice perspectiva que permita a quem observa determinado fenômeno se posicionar. Assim, a visão de mundo do pesquisador o estimula a problematizar a realidade que o cerca, sendo que isso pode ser feito por uma lente que o coloque como observador e sujeito que integra sua subjetividade a análise, ou ainda como observador afastado do fenômeno de pesquisa de forma objetiva. Essa relação muda a compreensão e o tipo de observação que se dará sobre um fenômeno, o que impactará diretamente nos resultados de uma pesquisa.

Palavras-chave: Ontologia. Epistemologia. Pesquisa. Metodologia.

\section{ABSTRACT}

The present conceptual article aimed to reflect on a coherent relationship between paradigm, methods and findings of a research. This article aims to encourage discussion with researchers, as well as editors and reviewers of academic journals. The discussion presented here follows in a heuristic manner a debate based on works that deal mainly with research in the social sciences area, focusing on the area of administration. The problem here is that the researcher first adopts a methodological approach as the position of his research. It is worth noting that the error lies in defending purely and simply a methodological strategy as if it were the research paradigm. Thus, a natural alignment would be to establish a relation of the research paradigm to a problematic, and from that to a methodological strategy. It is hoped that the discussion presented here will contribute knowledge that will be built based on a triple perspective that allows those who observe a particular phenomenon to position themselves. Thus, the world view of the researcher stimulates him to problematize the reality that surrounds him, and this can be done by a lens that places him as an observer and subject that integrates his subjectivity into the analysis; or as an observer away from the phenomenon of research objectively. This relationship changes the understanding and the type of observation that will be given about a phenomenon, which will directly impact the results of a research.

Keywords: Ontology; Epistemology; Search; Methodology. 


\section{INTRODUÇÃO}

A realidade do início do século XXI traz em seu bojo avanços tecnológicos em crescimento exponencial, além de problemas de ordem social e ambiental que exigem soluções transnacionais (KITCHEL, 2016; MORIN, 2013; STARK; JAKUBEK; KOBUS, 2015). Nesse contexto, o papel dos pesquisadores se torna cada vez mais importante, pois as explicações sobre os fenômenos e a busca por soluções para os problemas precisam se apresentar rapidamente. Essa realidade apresenta certo grau de complexidade que dificilmente é explicado por pensamentos isolados ou fragmentados. Um exemplo dessa complexidade é a corrida global para compreender e trazer soluções para o vírus da Zica.

Os indivíduos que possuem como objetivo pesquisar e trazer contribuições ao mundo acadêmico, bem como para a sociedade, precisarão expandir seu arsenal metodológico e sua maneira de pensar o mundo no que concerne a pesquisa no século XXI. Essa questão é aqui levantada porque muitas vezes o pesquisador fica limitado por muros paradigmáticos ou pressuposições enviesadas por tradições ou metodologias de pesquisa. Além disso, as discussões de ordem prática ficam muitas vezes enuviadas por métodos e técnicas que justificam o processo, mas que podem comprometer fortemente os achados de uma pesquisa.

Deste modo, este artigo visa construir uma reflexão a respeito de pesquisa que esteja amparada em métodos e técnicas, mas que também esteja adequada a visão de mundo do pesquisador. A discussão aqui apresentada está baseada na conformidade entre perspectivas ontológicas, epistemológicas e metodológicas. Do ponto de vista metodológico, esta discussão inclui a escolha de amostras, métodos de coleta e de análise de dados, além da apresentação dos resultados que seguem estratégias de pesquisa quantitativas e qualitativas.

Além disso, se faz necessário dizer que debates sobre se pesquisas qualitativas são melhores que pesquisas quantitativas não trazem benefícios ao avanço do conhecimento científico. Nastasi e Schensul (2005) salientam que pesquisadores estritamente quantitativos consideram os dados qualitativos como anedóticos, não-científicos e enviesados pelas opiniões de seus pesquisadores. Trotter (2012) cita que pesquisadores que adotam pesquisas qualitativas ou qualitativas acreditam que possuem as respostas corretas com relação aos melhores métodos ou estratégias de pesquisa. Todavia, a discussão quanto ao tipo de estratégias de pesquisa deve ocorrer após o reconhecimento dos posicionamentos ontológico e epistemológico, seguindo na sequência a respectiva problematização do fenômeno pesquisado, respeitando assim a visão de mundo do pesquisador e os objetivos da pesquisa para a construção do conhecimento.

Vale destacar que muitos pesquisadores seguem um roteiro de estratégias de pesquisa sem se atentar a validade e contribuições de seus achados. Entretanto, se esses pesquisadores adotarem uma atitude consciente e crítica com relação aos procedimentos e paradigmas de pesquisa, eles poderão gerar avanços importantes em suas áreas de conhecimento. O artigo de Guerra, Gomes e Silva Filho (2015) sobre estudos de caso na área de gestão pública encontra inconsistências em diversos artigos pesquisados, como descrição confusa da metodologia; descrição de resultados inconsistentes com a coleta e a análise de dados; falta de informação a respeito dos entrevistados; e falta de descrição das variáveis investigadas. Falaster, Ferreira e Canela (2016) em pesquisa sobre os motivos de rejeição de artigos em periódicos brasileiros assinalaram que um dos maiores problemas está na metodologia, sendo que os pesquisadores brasileiros possuem entre outras dificuldades a de relatar suas contribuições nos artigos. 
Teixeira, Nascimento e Carrieri (2012) salientam que a triangulação dos métodos observados em 174 artigos do campo de pesquisas em administração tem sido adotada principalmente no intuito de validação convergente, não implicando necessariamente conversações efetivas entre diferentes visões de mundo e de conhecimento científico. O que se observou nesse estudo foi a aplicação predominante de pesquisas em que prevalece em termos epistemológicos um paradigma positivista, o que em termos metodológicos representa uma postura nomotética. Östlund et al. (2011) defendem que a utilização de forma metafórica da triangulação metodológica auxilia na análise e discussão de resultados de forma eficaz em uma pesquisa. Essa triangulação metodológica pode facilitar a identificação clara de relação entre diferentes níveis de uma teoria, pois ajuda a descrever as relações lógicas entre os achados qualitativos e quantitativos e os conceitos teóricos de uma pesquisa. Isto pode ser feito tanto numa direção dos dados para teoria (grounded), ou via teoria buscando testar ou validar hipóteses.

Cabe ressaltar que a proposta deste artigo não é apresentar ou propor um modelo de avaliação de pesquisa, se pretende apresentar um caminho para a escolha do método de pesquisa, conforme o alinhamento entre visão de mundo do pesquisador, proposta de pesquisa e achados. Como contribuição desse artigo há uma pretenciosa intenção de informar os pesquisadores mais novos e provocar os mais experientes sobre o alinhamento entre o paradigma, a pesquisa e os resultados esperados. Pode parecer óbvio para alguns pesquisadores a proposta de buscar uma relação de coerência e convergência entre esses três elementos, mas essa questão deve ser melhor discutida para dirimir julgamentos e posições adotadas muitas vezes pela falta de informação. Assim, esse artigo se propõe a discutir com pesquisadores, além de editores e revisores de revistas acadêmicas.

Este artigo é dividido da seguinte forma: na primeira parte são apresentados o contexto de discussão e uma breve explanação sobre a evolução de pesquisas qualitativas e quantitativas. Na sequência descrevese a importância do alinhamento entre paradigmas e a pesquisa, mas propondo a mitigação de barreiras paradigmáticas no que compete a adoção de procedimentos de pesquisa. Na seção seguinte são descritos as principais características e papéis das abordagens qualitativas e quantitativas. Na sequência é discutida a relação entre o alinhamento entre paradigma e os achados da pesquisa. Por último são apresentados itens que dão suporte a qualidade de pesquisam nas abordagens qualitativa e quantitativa.

\section{CONFORMIDADE ENTRE PROPOSTA DE PESQUISA E AÇÃO DO PESQUISADOR PARA APRIMORAR OS ACHADOS DE PESQUISA}

Em uma busca na base de dados Proquest (http://search.proquest.com/index) com as palavras "qualitative research" e "quantitative research", sem utilizar nenhum tipo de filtro, forram obtidos dados impressionantes com relação ao número de publicações que passam de centenas na década de 1970 para centenas de milhares nos anos 2000 (Tabela 1). Os resultados dessa busca apontam que as pesquisas de forma geral aumentaram em ordem exponencial. É necessário ressaltar que pode haver uma sobreposição de pesquisas nas duas categorias apresentadas na tabela 1, pesquisa qualitativa e pesquisa quantitativa, mas ainda assim os números apresentados são bastante expressivos.

As pesquisas quantitativas evoluíram muito nas últimas décadas, principalmente impulsionadas por sistemas informatizados que permitiram trabalhar com uma grande quantidade de informações que não seria possível sem esses aparatos. Os big datas já são fontes de evidências que permitem mapear e compreender 
causas de doenças pelo mundo (vírus da Zica), o nível de satisfação de milhões de consumidores (Netflix) e tendências migratórias (desertificação).

Tabela 1: Publicações no Proquest

\begin{tabular}{ccccccc}
\hline Período & \multicolumn{2}{c}{ Pesquisa qualitativa } & \multicolumn{2}{c}{ Pesquisa quantitativa } & Total & \% de crescimento \\
\hline $1960-1969$ & 411 & $31 \%$ & 921 & $69 \%$ & 1.332 & \\
$1970-1979$ & 2.529 & $35 \%$ & 4.716 & $65 \%$ & 7.245 & $544 \%$ \\
$1980-1989$ & 6.370 & $39 \%$ & 9.853 & $61 \%$ & 16.223 & $224 \%$ \\
$1990-1999$ & 41.371 & $44 \%$ & 51.779 & $56 \%$ & 93.150 & $574 \%$ \\
$2000-2009$ & 180.225 & $41 \%$ & 261.941 & $59 \%$ & 442.166 & $475 \%$ \\
$2010-2017$ & 314.854 & $39 \%$ & 499.327 & $61 \%$ & 814.181 & $184 \%$ \\
\hline
\end{tabular}

Fonte: Proquest, 2017 (http://search.proquest.com/index)

Nota: O resultado apresentado traz artigos que têm "métodos qualitativos" e "métodos quantitativos" em suas palavraschaves e que é possível ter sobreposição de artigos.

Do mesmo modo, as pesquisas que utilizam a abordagem qualitativa também seguiram os avanços em relação a atribuição de transparência e rigor metodológico em seus processos, o que conferiu mais legitimidade e confiabilidade nesse tipo de pesquisa. Softwares como o Atlas.TI e o NVivo auxiliaram muito na análise dos dados qualitativos, o que permitiu que as discussões e resultados de pesquisas com essa abordagem ficassem mais acessíveis e transparentes (NASTASI; SCHENSUK, 2005).

Além disso, pode-se observar na Tabela 1 que pesquisas qualitativas acompanharam o aumento no número de pesquisas quantitativas, tendo um ligeiro aumento de percentual. O seu uso tem proporcionado contribuições importantes em diversos campos de conhecimento como a administração de empresas e economia. A evolução na aplicação dos métodos e técnicas da abordagem qualitativa fez com que fossem reduzidas as lacunas entre pesquisas qualitativa e quantitativa.

\section{EQUALIZANDO PARADIGMA DE PESQUISA COM O PROBLEMA DE PESQUISA}

Propõe-se neste artigo que o pesquisador deve se posicionar primeiramente com relação a visão de mundo a ser utilizada na realização da pesquisa, definindo assim um paradigma. Guba e Lincoln (1994) dizem que a escolha dessa visão está relacionada ao paradigma que representa um conjunto de crenças e princípios de visão do mundo e de suas partes. Os autores ainda explicam que a escolha do paradigma de pesquisa deve estar baseada em três questionamentos: i) questões ontológicas; ii) questões epistemológicas; iii) questões metodológicas. Assim, como destaca Creswell (2010, p.28) são estabelecidas "[...] as concepções como uma orientação geral sobre o mundo e sobre a natureza da pesquisa defendidas por um pesquisador".

A ontologia é o estudo do ser, a forma como é compreendido como as coisas são. O seu objetivo é entender a essência e a forma da realidade identificando se o ser é abstrato ou concreto. A ontologia define o ser em suas categorias fundamentais, o que é construído a partir de suas propriedades e dimensões (GRAY, 2012; GUBA; LINCOLN, 1994). A realidade do ser pode constituir-se a partir da sua própria existência como objeto e/ou fenômeno, ou ainda do entendimento do sujeito que o observa. Assim, o ser pode ser ontologicamente explicado por uma perspectiva realista ou relativista. Na primeira, a realidade do ser independe do sujeito, a existência do ser independe de percepções e construções mentais. Na segunda 
perspectiva são aceitas múltiplas realidades que são criadas pelo sujeito que observa tal objeto e/ou fenômeno (GUBA; LINCOLN, 1994).

A epistemologia está ligada a construção do conhecimento de forma científica, ela representa a natureza e origem do conhecimento sem a utilização de achismos, opiniões ou do senso comum. Bêrni e Fernandez (2012, p.65) afirmaram que:

[...] a busca por um método que pudesse assegurar o "conhecimento seguro" (episteme) em contraste com a "mera opinião" (doxa) remonta à Grécia antiga. Esse conhecimento seguro seria conseguido por meio da filosofia, segundo então se acreditava. Os métodos para alcançá-los perpassavam a maiêutica socrática (ou o método do "parto das ideias" por meio do diálogo), a dialética platônica (ou o método da oposição duas ideias contrárias, com o fito de se chegar a uma terceira, distinta das anteriores), chegando à analítica (ou lógica) aristotélica (o método dedutivo da teoria dos silogismos).

Assim, a epistemologia estuda a origem, a estrutura, os métodos e a validade do conhecimento (GODOI; BANDEIRA-DE-MELO; SILVA, 2010). Seguindo a perspectiva ontológica, a obtenção do conhecimento pode ser colocada numa abordagem objetivista ou subjetivista (VERGARA; CALDAS, 2005). Portanto, a partir de diferentes visões ontológicas e epistemológicas são gerados diferentes paradigmas de pesquisa, que podem ser reconhecidos como perspectivas para construir uma visão de mundo.

Os paradigmas servem para direcionar os métodos que serão empregados para investigar um determinado fenômeno. No Quadro 1 podem ser identificadas as visões sobre pesquisa de Guba e Lincoln (1994) para os seguintes paradigmas: positivista, pós-positivista, teoria crítica, e construtivismo.

Os paradigmas apresentados no Quadro 1 podem exemplificar como a visão de mundo impacta nas escolhas de estratégias metodológicas para observar e explicar determinado fenômeno. Cabe aqui destacar que outras descrições sobre paradigmas podem ser encontradas. Como pode ser visto em Morgan (1980), que expõem como visões da realidade social os paradigmas: humanista radical, estruturalista radical, interpretativista e funcionalista. Esses paradigmas são representações baseadas em dois eixos: dimensão da natureza da ciência, que classifica a realidade como objetiva ou subjetiva; e dimensão da mudança social, que é explicada tendo nas suas extremidades a sociologia da regulação e a sociologia da mudança radical. Portanto, ao adotar uma perspectiva para analisar determinado fenômeno, consequentemente, chega-se a uma escolha metodológica.

Dessa forma, pode-se dizer que a metodologia é um ramo da epistemologia que representa o caminho para chegar ao conhecimento científico. Godoi, Bandeira-De-Melo e Silva (2010) explicam que a metodologia no processo de pesquisa é uma etapa secundária. A função da metodologia é delimitar os procedimentos que conduzirão a investigação como forma de coleta de dados e interpretação dos resultados. Vale destacar que a metodologia segue duas direções procedimentais, sendo uma realizada a partir das experiências pela observação livre dos fenômenos para chegar a proposições gerais, que é denominada de indutiva ou empirismo (theory building). Já de outra forma os procedimentos são conduzidos a partir de alegações gerais ou universais para realizar observações em particular de forma dedutiva (theory testing). Neste último tipo de procedimento surgem proposições que são reconhecidas como hipóteses, que podem ser testadas para validar o conhecimento obtido (BÊRNI; FERNANDEZ, 2012; GRAY, 2012). 
Quadro 1. Visões sobre pesquisa para quatro paradigmas

\begin{tabular}{|c|c|c|c|c|}
\hline & Positivismo & Pós-positivismo & Teoria Crítica & Construtivismo \\
\hline Ontologia & $\begin{array}{l}\text { Realismo ingênuo } \\
\text { - realidade "real", } \\
\text { mas apreensível. }\end{array}$ & $\begin{array}{l}\text { Realismo crítico - } \\
\text { realidade "real", } \\
\text { mas apenas } \\
\text { imperfeitamente e } \\
\text { probabilisticamente } \\
\text { apreensível. }\end{array}$ & $\begin{array}{l}\text { Realismo histórico, } \\
\text { realidade virtualmente } \\
\text { moldada pelo social, } \\
\text { Político, cultural, } \\
\text { econômico, étnico e de } \\
\text { gênero; conhecimento } \\
\text { cristalizado ao longo do } \\
\text { tempo. }\end{array}$ & $\begin{array}{l}\text { Relativismo- } \\
\text { local e } \\
\text { realidades } \\
\text { específicas } \\
\text { construídas. }\end{array}$ \\
\hline Epistemologia & $\begin{array}{l}\text { Dualista/ } \\
\text { objetivista; } \\
\text { Achados } \\
\text { verdadeiros }\end{array}$ & $\begin{array}{l}\text { Dualista } \\
\text { modificada / } \\
\text { objetivista; } \\
\text { Tradição crítica / } \\
\text { comunidade; } \\
\text { Achados } \\
\text { provavelmente } \\
\text { verdadeiros. }\end{array}$ & $\begin{array}{l}\text { Transacional / Subjetivista; } \\
\text { Resultados mediados pelo } \\
\text { valor. }\end{array}$ & $\begin{array}{l}\text { Transacional / } \\
\text { Subjetivista; } \\
\text { Descobertas } \\
\text { criadas. }\end{array}$ \\
\hline Metodologia & $\begin{array}{l}\text { Experimental / } \\
\text { manipulativo; } \\
\text { Verificação de } \\
\text { hipóteses; } \\
\text { Principalmente } \\
\text { métodos } \\
\text { quantitativos. }\end{array}$ & $\begin{array}{l}\text { Experimentos } \\
\text { modificados / } \\
\text { Manipulativo; } \\
\text { Multiplicismo } \\
\text { crítico; Verificação } \\
\text { de Hipóteses; } \\
\text { Podem incluir } \\
\text { métodos } \\
\text { qualitativos. }\end{array}$ & Dialógico / dialética & $\begin{array}{l}\text { Hermenêutico / } \\
\text { Dialético }\end{array}$ \\
\hline
\end{tabular}

Fonte: adaptado de Guba e Lincoln (1994).

Sauerbronn e Ayrosa (2010) defendem em seu artigo a importância do posicionamento epistemológico para estudar determinado fenômeno. Os autores ainda dizem que devem ser usados métodos qualitativos com orientação construtivista-interpretativista para identificar e entender símbolos e significados de um fenômeno. Vale destacar que a adoção de um posicionamento ontológico e epistemológico durante uma pesquisa determina a escolha de procedimentos e técnicas que podem enviesar os seus resultados (DENZIN; LINCOLN, 2006).

Portanto, ao entender a perspectiva ontológica e epistemológica usadas para conduzir a pesquisa, além de alinhá-las com as escolhas dos métodos, pode-se melhorar a relação entre pesquisadores e avaliadores, ou mesmo entre pesquisadores. Creswell e Miller (2000) reforçam que pesquisadores póspositivistas escolhem métodos mais estruturados e sistematizados. Já os pesquisadores construtivistas selecionam métodos que privilegiam a investigação em profundidade dos fenômenos, acima da rigidez na estruturação metodológica. Já pesquisadores que escolhem um paradigma crítico buscam a validação e colaboração constante dos participantes da pesquisa.

Contudo, neste artigo é defendido que o paradigma mais confortável para o pesquisador não deve impedir o uso de outros, pois caso contrário o pesquisador passará a procurar somente explicações para fenômenos que lhe convier. Além disso, pode-se dizer que quando não se amplia a perspectiva de análise, 
perde-se a oportunidade de enxergar incidentes relevantes na coleta e análise dos dados. Um pesquisador pode descartar o poder de missing values ou outliers, perdendo a oportunidade de uma investigação mais aprofundada e cuidadosa desse tipo de informação que poderia levar a achados importantes.

Outro aspecto que deve ser explicitado é que os métodos dedutivos geralmente utilizam estratégias de pesquisa com a abordagem quantitativa, já métodos indutivos usam estratégias de pesquisa com abordagem qualitativa. Porém, mesmo em estudos que adotem a abordagem qualitativa, pode-se afirmar que o viés por um paradigma de pesquisa leva o pesquisador a descartar incidentes ou observações importantes. O motivo deste posicionamento ocorre porque ele busca muitas vezes encontrar um padrão nos dados que foi definido inicialmente, o que o leva a desprezar um outlier que pode contribuir para explicar comportamentos e/ou fenômenos. Assim, o tom de voz, uma pausa, ou a própria falta de resposta podem ser mais importantes do que uma resposta objetiva e explicita.

Por mais polêmico que possa parecer, defende-se aqui a utilização de pesquisas quantitativas sob uma perspectiva interpretativista, aberta, plural e livre de ideologismos, ou outros "ismos". Yanchar (2006) diz que os pesquisadores devem proceder sem viés ou barreiras na construção de bases de dados. As fontes de dados podem ser encontradas de forma interpretativa e traduzidas numericamente a partir de textos, entrevistas, artefatos, observações, fotos, mapas e experiências de todos os tipos. Isto pode ser feito pela análise de frequência ou pela categorização dos incidentes numericamente em escalas ou adotando uma relação binária (SANDELOWSKI, 2000; SWEENEY, WILLIAMS; ANDERSON, 2013).

Portanto, diferentemente de pesquisas que adotem o método dedutivo e estratégias quantitativas, na pesquisa qualitativa pode-se dizer que sua ação permite estudar os fenômenos com uma maior proximidade, numa interação entre pesquisador e objeto de pesquisa. Além disso, na abordagem qualitativa é permitida a utilização de paradigmas diferentes e técnicas de análise no processo de investigação, como no método de análise de conteúdo de Bardin (2009) que utiliza análise de frequência.

\section{PESQUISADOR E ABORDAGEM DE PESQUISA}

Como visto na seção anterior, a perspectiva paradigmática adotada em uma pesquisa pode determinar o equilíbrio entre os objetivos da pesquisa e os procedimentos metodológicos. Uma pesquisa que possui como objetivo compreender determinado comportamento social pode adotar tanto procedimentos quantitativos, quanto procedimentos qualitativos. Contudo, o que realmente muda com relação a essa compreensão é o tipo de observação que se dará sobre o fenômeno, o que impactará diretamente nos resultados da pesquisa.

Desse modo, a discussão quanto a melhor estratégia de pesquisa não deve ser pessoal nem determinística, pois alguns pesquisadores baseiam suas escolhas sobre uma ou outra abordagem com um posicionamento puramente ideológico. Denzin e Lincoln (2006, p. 22) destacaram que a pesquisa qualitativa é vista muitas vezes como um trabalho "[...] não-científico, ou apenas exploratório, ou subjetivo. É chamado de crítica, e não de teoria, ou é interpretado politicamente como uma versão disfarçada do marxismo ou do humanismo secular." Por outro lado, Westerman (2006) discute o quanto interpretativa pode ser uma pesquisa com abordagem quantitativa em pesquisas sobre interações sociais. 
Como defendem Creswell (2010) e Trotter (2012), a abordagem qualitativa tem produzido teorias cientificamente robustas com evidente rigor metodológico. Além disso, pesquisas que adotam métodos mistos potencializam os pontos fortes e minimizam as limitações de cada abordagem.

Westerman (2011) defende que a escolha do método, ou caminho metodológico, dependerá da forma como a pergunta de pesquisa precisa ser respondida. Assim, o problema de pesquisa orienta a estratégia de pesquisa de tal forma que os instrumentos de coleta e análise serão escolhidos coerentemente com os objetivos da pesquisa. Rich e Ginsbug (1999) e Yin (2008) descrevem que questões básicas como "O que?", "Quando?" "Seja ou não?", "Quantos?", entre outras, são mais afins de uma abordagem quantitativa por permitir respostas concretas e objetivas. Já questões que são compostas por "Como?", "Por quê?", "O que causa?", entre outras, podem utilizar uma abordagem qualitativa para procurar respostas, bem como construir hipóteses.

Vale destacar que a hipótese é outra controvérsia quando se discutem as duas abordagens. Muitos pesquisadores defendem que a pesquisa qualitativa não parte de hipóteses, talvez pressupostos, mas que tem como um de seus objetivos a elaboração de hipóteses (CRESWELL, 2010; BÊRNI; FERNANDEZ, 2012; GRAY, 2012).

Minayo (2010) afirma que a função da pesquisa qualitativa é a de buscar significados, motivos, aspirações, crenças, valores e atitudes. A autora defende que o universo de significar está presente no processo de interações com o fenômeno, que não pode estar reduzida a operacionalização de variáveis. A pesquisa qualitativa constrói uma espécie de artefato heurístico de construção da realidade. É preciso compreender e interpretar dentro do contexto do fenômeno, o que exige uma reflexão crítica do observador e do observado.

O pesquisador que utiliza a abordagem qualitativa possui uma relação de interdependência com o fenômeno e/ou objeto pesquisados. Os indivíduos que fornecem informações para explicar dado fenômeno só podem ser representados por meio do pesquisador, sendo esse por sua vez instrumento de coleta e análise, além de porta voz dos resultados. Pode-se dizer que a subjetividade do pesquisador está presente em todo o processo de pesquisa. Como descrevem Denzin e Lincoln (2006), na pesquisa qualitativa o pesquisador utiliza um conjunto de práticas interpretativas que dão visibilidade ao mundo transformando-o em uma série de representações, que podem ser evidenciadas nas notas de campo, nas entrevistas, nas conversas, nas imagens, entre outras fontes de evidências utilizadas para compreender e interpretar os fenômenos em termos de significados.

Westerman (2006) informa que não há um grau de subordinação entre pesquisas qualitativas ou quantitativas, mas que elas são acessórias. Não obstante, Westerman (2011) reforça o desconforto que é causado aos pesquisadores interpretativistas quando eles são obrigados a buscar ferramentas quantitativas para auxiliar em seus trabalhos. Todavia, os métodos quantitativos podem se mostrar úteis em situações que exige certos tipos de comparações sistemáticas, o que permite uma exploração mais objetiva sobre o objeto e o fenômeno analisados (WESTERMAN, 2011). No Quadro 2 são expostas as principais características de cada abordagem metodológica.

Silverman (2009) destaca que uma aproximação de pesquisas qualitativas com pesquisas quantitativas pode possibilitar complementaridade entre os métodos, além de contribuir com a apresentação de resultados que representem importantes avanços na ciência. Desse modo, a decisão de utilizar métodos 
quantitativos e/ou qualitativos dependerá dos objetivos da pesquisa, bem como das características específicas do fenômeno estudado. Vale lembrar que a forma indutiva de analisar os dados está mais alinhada com a pesquisa qualitativa, já o direcionamento para formas dedutivas, com base em testes de hipóteses, segue uma abordagem quantitativa (CRESWELL; MILLER, 2000; NASTASI; SCHENSUK, 2005).

Quadro 2: Diferenças entre design qualitativo e quantitativo

\begin{tabular}{|c|c|}
\hline Qualitativa & Quantitativa \\
\hline Culturalmente representativa & Individualmente representativa \\
\hline Framework especialista & Framework probabilístico \\
\hline Modelo de saturação & Modelo estatístico \\
\hline Generalização analítica & Generalização estatística \\
\hline Foco no consenso com as variações descritas & $\begin{array}{c}\text { Foco na variação, tendência central e representação } \\
\text { estatística }\end{array}$ \\
\hline
\end{tabular}

Fonte: adaptado de Trotter (2012).

Um ponto que necessita ser debatido com relação as pesquisas qualitativas e quantitativas é quanto ao processo de dada uma. Um pesquisador que segue a estratégia quantitativa possui roteiros e padrões mais racionais para conduzir sua pesquisa. Ele pode acompanhar modelos a partir das hipóteses até à análise e apresentação dos resultados. Além disso, parâmetros são estabelecidos para que sejam gerados os indicadores de validade e confiabilidade do estudo (SWEENEY; WILLIAMS; ANDERSON, 2013). Um exemplo desse fato é o artigo "Modelagem de equações estruturais com utilização do SmartPLS" de Ringle, Silva e Bido (2014). Nesse artigo são encontradas todas as etapas e orientações para realizar coleta dos dados e análise e apresentação dos resultados. Assim, na pesquisa quantitativa pode-se escolher o grau de confiabilidade e determinar a amostra como visto no citado artigo.

Nas estratégias qualitativas não existe uma forma rígida de se fazer pesquisa, os fenômenos podem ser estudados combinando teorias existentes com Grounded Theory. A pesquisa pode começar com um modelo teórico para orientar a coleta de dados, mas também pode adotar métodos mais abertos como os estudos etnográficos. Assim, após a análise dos dados podem ser produzidos resultados antecipados pela revisão sistemática da literatura, bem como resultados inesperados que emergem dos dados (NASTASI; SCHENSUK, 2005; DENZIN; LINCOLN, 2006). Como destacaram Nastasi e Schensuk (2005), a pesquisa qualitativa pode seguir uma orientação dedutiva-indutiva em seu processo. Na abordagem qualitativa o processo que o pesquisador deve seguir é mais flexível (MARSHAL; ROSSMAN, 2006). Entretanto, Nastasi e Schensuk (2005) afirmam que o pesquisador deve fazer um planejamento prévio, com detalhes específicos e concretos, de forma a mostrar a ética adotada, a mitigação dos vieses e as mudanças efetuadas nesse planejamento durante o percurso da pesquisa.

Uma forma de diferenciar o processo de métodos quantitativos e qualitativos é que os primeiros trabalham com dados discretos, numéricos, analisando suas frequências e associações estatísticas, enquanto os métodos qualitativos observam narrativas, significados e comportamentos no contexto social (RICH; GINSBURG, 1999; MINAYO, 2010). Na pesquisa quantitativa, os pesquisadores observam o fenômeno de fora, sem se envolver com o objeto de pesquisa. Eles fazem uso de variáveis objetivas e tangíveis de forma 
dedutiva com baixo ou nenhum grau de subjetividade. Os valores numéricos que são atribuídos ao estudo garantem sua validade por meio de testes estatisticamente significativos (RICH; GINSBURG, 1999). Já na utilização de métodos qualitativos a perspectiva utilizada para estudar é de dentro para fora, de forma indutiva, na qual os pesquisadores observam de maneira interativa os comportamentos evidenciados a partir das percepções humanas, relações e crenças (MARSHAL; ROSSMAN, 2006).

Vale ressaltar que os aspectos relacionados à subjetividade e ao envolvimento do pesquisador fazem com que a coleta de dados, a análise e apresentação dos resultados se tornem mais complexos. Isso não quer dizer que os métodos quantitativos sejam mais fáceis. O que está sendo dito aqui é que mesmo com a existência de roteiros para a realização de coleta e tratamento de dados, o método qualitativo depende do pesquisador como participante e sujeito de pesquisa. Logo, o pesquisador desempenha o papel de instrumento de coleta e análise na pesquisa qualitativa, sendo esse um dos principais pontos de polémica na discussão entre as abordagens qualitativa e quantitativa. O motivo dessa polêmica é o grau de subjetividade e envolvimento do pesquisador nas inferências na pesquisa qualitativa (NASTASI; SCHENSUK, 2005; DENZIN; LINCOLN, 2006).

Desse modo, o questionamento a respeito das pesquisas qualitativas por parte da denominada ciência natural se baseia na exigência de que o conhecimento obtido de determinado fenômeno deva ser quantificado para ter validade (DENZIN; LINCOLN, 2006; BÊRNI; FERNANDEZ, 2012). No entanto, o conhecimento gerado a partir da pesquisa qualitativa parte da interpretação, tendo o pesquisador como seu instrumento de pesquisa na análise e interpretação de textos, sons, imagens, linguagem corporal, entre outras formas que possam servir de fonte de evidência como a comunicação não-verbal dos indivíduos (GUBA; LINCOLN, 1994; MINAYO, 2010). Além disso, o pesquisador qualitativo trabalha frequentemente com um número pequeno de amostras, estudando-as em profundidade para obter informações detalhadas sobre os participantes ou informantes (RICH; GINSBURG, 1999; MARSHAL; ROSSMAN, 2006).

Embora as pesquisas quantitativas e qualitativas sejam vistas como opostas por muitos pesquisadores, como salienta Rich e Ginsburg (1999) e Östlund et al. (2011), essa polarização é artificial e imprecisa. Esses autores reforçam o debate até aqui exposto, dizendo que pesquisadores quantitativos avaliam a pesquisa qualitativa como carente de rigor científico. De outro lado, os pesquisadores qualitativos acreditam que as pesquisas quantitativas não levam em conta a subjetividade dos indivíduos. Contudo, como defendem Sandelowski (2000) e Creswell (2010) a utilização de estudos que adotem métodos mistos é uma realidade que não pode ser negada, mesmo porque estratégias complementares permitem maior exploração do fenômeno, bem como robustez na construção de novas teorias.

\section{ACHADOS DA PESQUISA}

Como foi abordado nas seções anteriores deste artigo, o paradigma de pesquisa ou as escolhas metodológicas muitas vezes enviesam os estudos dos pesquisadores. Contudo, não deve ser um problema quando o posicionamento está relacionado a sua visão de mundo. O posicionamento paradigmático faz com um pesquisador estabeleça a forma com que enxerga os sinais e os artefatos de dado fenômeno (conteúdo). Além disso, pesquisadores que adotam um paradigma crítico tenderão a observar questões que se 
proponham a emancipação dos participantes da pesquisa, numa relação interdependente entre pesquisador e o sujeito de pesquisa.

Desse modo, destaca-se que o problema aqui evidenciado é o pesquisador adotar primeiramente como posicionamento de sua pesquisa o método. O erro está em defender pura e simplesmente o método como se este fosse o paradigma de pesquisa. Assim, o correto é estabelecer uma relação do paradigma de pesquisa para uma pergunta, e da pergunta para o método. Como salientam Creswell e Miller (2000), pesquisadores que posicionam sua pesquisa como pós-positivista, interpretativismo, construtivismo social ou a teoria crítica costumam seguir estratégias qualitativas, principalmente os pós-positivista utilizam as estratégias qualitativas de maneira complementar as estratégias quantitativas.

Portanto, tanto pesquisas qualitativas quanto pesquisas quantitativas estão sujeitas ao enviesamento do paradigma de pesquisa. Assim, o entendimento do posicionamento ontológico e epistemológico de um pesquisador pode fazer com que ocorram escolhas por coletar um dado que contribui para uma análise estatística sofisticada, mas que não contribui para o avanço da ciência (KING, 1986). Essa situação se apresenta como uma realidade em estratégias quantitativas quando o pesquisador está mais preocupado com a técnica do que com o problema de pesquisa. Cabe ressaltar que o objetivo aqui não é depreciar as análises estatísticas, mas explorar a importância de seu uso, buscando explicitar sua função no estudo de determinado fenômeno (WESTERMAN, 2006; WESTERMAN, 2011). Isto posto, pode-se dizer que a clareza e objetividade das estratégias quantitativas faz com que o processo se torne mais importante para pesquisadores incautos, do que o resultado para a sociedade.

Nastasi e Schensul (2005) dizem que em contraste com a abordagem dedutiva conduzida pela hipótese na pesquisa quantitativa, a pesquisa qualitativa geralmente reflete um processo emergente impulsionado por questões de pesquisa e interações entre pesquisador e objeto em seu contexto natural. Assim, a pesquisa qualitativa pode gerar hipóteses com base no significado e modelos teórico-conceituais que são gerados a partir de uma realidade dialógica. Como descreve Morin (1977, p. 80) "[...] digamos que dialógico significa unidade simbiótica de duas lógicas, que simultaneamente se alimentam uma à outra, se concorrenciam, se parasitam mutuamente, se opõem e se combatem mortalmente [...]". Na sequência Morin ainda explica que "[...] a ideia de dialógico situa-se ao nível do princípio e, como ouso adiantar, ao nível do paradigma [...]". No processo dialógico é possível compreender a intersubjetividade que surge não da declaração dos participantes da pesquisa, mas do diálogo e da confrontação.

Deste modo, ao concluir uma pesquisa alinhando propostas ontológicas, epistemológica e metodológica se pode garantir maior robustez aos achados de uma pesquisa. O motivo para essa afirmação está no fato de que se há uma proposta de analisar sob uma perspectiva crítica determinado fenômeno, o pesquisador buscará significados no discurso e no comportamento dos sujeitos pesquisados. Assim, independente da quantidade ou da abrangência explicativa, a pesquisa se tornará importante para seus pares uma vez que apresente uma discussão em que o sujeito e o fenômeno estejam integrados e representados no discurso do pesquisador. Por outro lado, em uma perspectiva positivista ocorrerá o afastamento do pesquisador do sujeito de pesquisa, bem como sua objetividade na análise e interpretação dos achados. 


\section{Qualidade da pesquisa}

Um ponto que merece ser apresentado na discussão proposta neste artigo é a questão da qualidade dos achados de uma pesquisa. Os critérios que garantem que uma pesquisa seja considerada relevante e rigorosa com relação às abordagens qualitativas e quantitativas são diferentes. Lincoln e Guba (1985) propuseram um conjunto de critérios para avaliar a pesquisa qualitativa a fim de garantir confiabilidade aos seus resultados (trustworthiness), são eles: a) credibilidade; b) transferibilidade; c) dependabilidade; d) confirmabilidade. Vale reforçar que os critérios de qualidade na pesquisa qualitativa são aqueles que asseguram a validade e a confiabilidade. O Quadro 3 apresenta a relação entre os critérios da pesquisa qualitativa com os critérios da pesquisa quantitativa.

Quadro 3: Critérios de confiabilidade em pesquisa

\begin{tabular}{|c|c|}
\hline Pesquisa qualitativa & Pesquisa quantitativa \\
\hline Credibilidade & Validade interna \\
\hline Transferibilidade & Validade externa \\
\hline Dependabilidade & Confiabilidade \\
\hline Confirmabilidade & Objetividade \\
\hline
\end{tabular}

Fonte: adaptado de Lincoln e Guba (1985)

Vale destacar que na pesquisa qualitativa a credibilidade está relacionada à confiança na "verdade" dos achados. Lincoln e Guba (1985) afirmam que a confiabilidade de um estudo de pesquisa garante credibilidade aos seus achados. A validade interna na pesquisa quantitativa se refere à capacidade que os métodos utilizados numa pesquisa propiciam a realização dos objetivos. Kirk e Miller (1986) dizem que na tradição qualitativa, a validade tende a ser observada nos seguintes aspectos: validade aparente, que é alcançada quando um método de pesquisa produz o tipo de informação desejado ou esperado; validade instrumental, que procura a combinação entre os dados fornecidos por um método de pesquisa e aqueles gerados por algum procedimento alternativo, que é aceito como válido; e validade teórica, que se refere à legitimidade dos procedimentos da pesquisa em termos de teoria estabelecida.

A transferibilidade representa o estado em que a pesquisa permite que os seus resultados sejam aplicados em outros contextos. A generalização na pesquisa quantitativa é um objetivo claro, mas quando o assunto é pesquisa qualitativa os objetivos principais são explorar e significar (POLIT; BECK, 2010). Contudo, do mesmo modo que na pesquisa quantitativa a generalização é realizada na pesquisa qualitativa pela transferibilidade, pois padrões são identificados e podem explicar alguns comportamentos que são produzidos a partir de determinadas situações.

A dependabilidade demonstra que os achados são consistentes e podem ser repetidos. Por sua vez, a confiabilidade refere-se à garantia de que outros pesquisadores conseguirão realizar uma pesquisa semelhante e chegar a resultados aproximados (KIRK; MILLER, 1986). Na pesquisa quantitativa os resultados de um pesquisador podem ser facilmente testados por outro pesquisador, o teste de um modelo conceitualteórico é conduzido com maior clareza e objetividade. Já a replicação do estudo na pesquisa qualitativa é uma questão controvérsia, principalmente porque leva a discutir não somente o tamanho da amostra, mas 
também por compartilhar uma visão da realidade do pesquisador que poderá ser assimilada e utilizada ou não por outros pesquisadores. Como dizem Polit e Beck (2010) pesquisadores que utilizam a abordagem qualitativa selecionam a amostra de pesquisa visando deliberadamente a replicação de seu estudo, o que permite a generalização analítica e a transferibilidade. Contudo, fica aqui uma pergunta: Como os resultados de uma pesquisa qualitativa podem ser generalizados? Desse modo, outra questão que aparentemente não é relevante em pesquisas qualitativas se torna evidente, A quantidade importa?

Nastasi e Schensul (2005) defendem que o universo amostral na pesquisa qualitativa não deve ser uma restrição. A amostra é proposital e muitas vezes incontrolável, uma vez que o pesquisador utiliza outras métricas para avaliar a validade e confiabilidade de seus resultados. $O$ que se mostra diferente quando o assunto são pesquisas orientadas para testar hipóteses numa perspectiva dedutiva com o uso de estratégias quantitativas. Polit e Beck (2010) dizem que a validade externa, ou seja, o grau em que as inferências de um estudo podem ser generalizáveis se tornou um padrão valioso na avaliação de trabalhos acadêmicos. Os autores apresentam um questionamento muito relevante cuja generalização se dá por conveniência numa atividade idiográfica, pois requer extrapolação que nunca pode ser totalmente justificada porque os resultados estão sempre introduzidos em um contexto específico. Todavia, se adotada a perspectiva de que alcançadas determinadas condições podem ser gerados comportamentos específicos, assim pode-se dizer que haverá uma extrapolação por explicar determinado comportamento em condições especificas. Vale destacar que essa é uma generalização diferente da estatística (CRESWELL, 2010).

Mason (2010) diz que embora existam outros fatores que afetem a avaliação da amostra em pesquisas qualitativas, os pesquisadores utilizam a saturação como viés para seleção da amostra. Apesar da ampla discussão sobre o tamanho da amostra, muitos pesquisadores ainda ficam indecisos ou inseguros na determinação de um número que garanta validade e confiabilidade na pesquisa. Guest, Bunce e Johnson (2006) sugerem que a ideia de saturação é muito útil no nível conceitual, pois garante uma orientação prática para determinar o tamanho da amostra. Assim, pode-se dizer que muitos pesquisadores possuem como objetivo encontrar uma relação entre os resultados da amostra com uma população a fim de explicar determinado comportamento, ou seja, a generalização teórica.

Firestone (1993) apresentou uma tipologia que descreve três formas de generalização que podem ser adotados para os achados de uma pesquisa. O primeiro tipo foi reconhecido como a extrapolação de uma amostra para uma população (generalização estatística), sendo esta a forma mais reconhecida de generalização que dá suporte aos estudos quantitativos. O segundo tipo de generalização é a analítica, sendo este um modelo que pode auxiliar tanto em pesquisas com abordagem qualitativa quanto estratégias quantitativas. O modelo analítico é baseado numa perspectiva em que são constituídas algumas pressuposições que permitem generalizações em relação entre casos e teorias.

O terceiro modelo é a transferência caso-a-caso na qual determinados fatores que não seriam explorados, se dependessem da população, são importantes para pesquisas qualitativas que objetivam uma explicação em particular (SCHWANDT, 1997). A transferência caso-a-caso ocorre sempre que uma pessoa em um ambiente considera a adoção de um comportamento do outro. Um exemplo desse modelo de generalização é o estudo de Allison (1971) sobre a crise dos mísseis cubanos, pois o autor usou um evento dramático para comparar três teorias proeminentes sobre tomada de decisão. 
A confirmabilidade demonstra a imparcialidade do pesquisador, o grau de neutralidade ou de influência com que os resultados de um estudo são moldados pelos entrevistados e não pelos interesses dos pesquisadores (LINCOLN; GUBA 1985). Nas pesquisas que seguem estratégias quantitativas este ponto é semelhante, a objetividade garante a validade e confiabilidade nas observações dos pesquisadores que asseguram legitimidade para os resultados gerados.

\section{CONSIDERAÇÕES FINAIS}

Ao final desse estudo pode-se depreender que o conhecimento é construído com base em uma tríplice perspectiva que permite a quem observa determinado fenômeno se posicionar. Para tanto, um posicionamento ontológico e epistemológico leva a uma escolha paradigmática sobre a realidade observada. Desse modo, as duas primeiras perspectivas, ontológica e epistemológica, deveriam conduzir a escolhas metodológicas coerentes. A visão de mundo do pesquisador o estimula a problematizar a realidade que o cerca por uma lente que o coloque como observador e sujeito, ou como observador afastado do fenômeno.

A principal contribuição desse artigo está na reflexão a respeito da conformidade entre proposta de pesquisa, pesquisador e achados. A questão sobre se a pesquisa deve ser quantitativa ou qualitativa é secundária, pois se não estiverem claros os aspectos ontológicos e epistemológicos da pesquisa o método pode ser somente uma escolha conveniente e pessoal. É necessário reforçar que a escolha entre estratégias que empreguem a abordagem quantitativa ou qualitativa, não deve ser definida somente pelo conhecimento existente sobre métodos ou conformidade na visão de mundo do pesquisador. Além disso, cabe relembrar que numa visão pós-positivista, os métodos mistos podem representar uma opção importante para promover avanços na ciência.

Um aspecto importante que deve ser ressaltado é que infelizmente os procedimentos metodológicos são escolhidos muitas vezes com base em um viés ideológico. A escolha entre estratégias qualitativas e quantitativas passa por questões pessoais, assim se esquece de que de um lado busca-se mensurar e do outro significar a realidade estudada. Em essência cada abordagem metodológica possui sua função. A pesquisa quantitativa objetiva a aplicação de técnicas para testar de maneira dedutiva hipóteses. Já a pesquisa qualitativa possui como principal objetivo compreender e interpretar significados observados das intencionalidades dos indivíduos durante as interações sociais.

Vale ainda destacar que não há nenhum grau de subordinação entre essas duas abordagens. Aliás, o que deve orientar as estratégias metodológicas é o objetivo da pesquisa, pois esse representa a principal forma de responder ao problema de pesquisa. Portanto, a discussão proposta nesse artigo instiga um olhar crítico a partir da problematização, coleta e análise dos dados, sendo necessário ter em mente que um paradigma de pesquisa não é somente uma categorização. A escolha ou identificação de posições ontológicas e epistemológicas justificam procedimentos e resultados que ora buscam significar fenômenos de forma dialógica, ora buscam testar modelos estatísticos. 


\section{REFERÊNCIAS}

ALLISON, Graham Tillett. Essence of decision: Explaining the Cuban missile crisis. Boston: Little, Brown, 1971.

BARDIN, Lawrence. Análise de conteúdo. Lisboa: Edições, 70, 2009.

BERNI, Duilio de Avila; FERNANDEZ, Brena Paula Magno. Métodos e técnicas de pesquisa-modelando as ciências empresariais. São Paulo: Saraiva, 2012.

CRESWELL, John W.; MILLER, Dana L. Determining validity in qualitative inquiry. Theory into practice, v. 39, n. 3, p. $124-$ 130, 2000.

CRESWELL, John W. Projeto de pesquisa métodos qualitativo, quantitativo e misto. Porto Alegre: Artmed, 2010.

DENZIN, Norman K; LINCOLN, Yvonna S. O planejamento da pesquisa qualitativa: teorias e abordagens. Porto Alegre: Artmed, 2006.

FALASTER, Christian; FERREIRA, Manuel Portugal; CANELA, Renata. Motivos de rejeição dos artigos nos periódicos de Administração. Organizações \& Sociedade, v. 23, n. 77, p. 285-306, 2016.

FIRESTONE, William A. Alternative arguments for generalizing from data as applied to qualitative research. Educational researcher, v. 22, n. 4, p. 16-23, 1993.

GODOI, Christiane Kleinübing; BANDEIRA-DE-MELLO, Rodrigo; SILVA, AB da. Pesquisa qualitativa em estudos organizacionais: paradigmas, estratégias e métodos. São Paulo: Saraiva, 2010.

GRAY, David E. Pesquisa no mundo real. Porto alegre: Penso Editora, 2012.

GUBA, Egon G.; LINCOLN, Yvonna S. Competing paradigms in qualitative research. In DENZIN, N. K.; LINCOLN, Y. S. (Eds.), Handbook of qualitative research (pp. 105-117). Thousand Oaks, CA: Sage, 1994

GUERRA, Mariana; GOMES, Adalmir de Oliveira; SILVA FILHO, Antônio Isidro da. Case study in public administration: a critical review of Brazilian scientific production. Revista de Administração Contemporânea, v. 19, n. 2, p. $270-289,2015$.

GUEST, Greg; BUNCE, Arwen; JOHNSON, Laura. How many interviews are enough? An experiment with data saturation and variability. Field methods, v. 18, n. 1, p. 59-82, 2006.

KING, Gary. How not to lie with statistics: Avoiding common mistakes in quantitative political science. American Journal of Political Science, v. 30, n. 3, p. 666-687, 1986.

KIRK, Jerome; MILLER, Marc L. Reliability and validity in qualitative research. Thousand Oaks: Sage Publications, 1986. KITCHEL, David. A real and monetary analysis of capitalism. Journal of Evolutionary Economics, v. 26, n. 2, p. 443-464, 2016.

LINCOLN, Yvonna S.; GUBA, Egon G. Naturalistic inquiry. Thousand Oaks, CA: Sage, 1985.

MASON, Mark. Sample size and saturation in PhD studies using qualitative interviews. In: Forum qualitative Sozialforschung/Forum: qualitative social research, v. 11, n. 3, 2010.

Minayo, MC De Souza. O desafio do conhecimento: pesquisa qualitativa em saúde. 12. ed. São Paulo: Hucitec, 2010.

MARSHALL, Catherine; ROSSMAN, Gretchen B. Designing qualitative research. 4 Ed. Thousand Oaks: Sage publications, 2006.

MORGAN, Gareth. Paradigms, metaphors, and puzzle solving in organization theory. Administrative science quarterly, p. 605-622, 1980.

MORIN, Edgard. O método: 1 - A natureza da natureza. $2^{\circ}$ ed. Sintra: Publicações Europa-América, 1977.

MORIN, Edgar. A via. São Paulo: Bertrand, 2013.

NASTASI, Bonnie K.; SCHENSUL, Stephen L. Contributions of qualitative research to the validity of intervention research. Journal of School Psychology, v. 43, n. 3, p. 177-195, 2005.

ÖSTLUND, Ulrika et al. Combining qualitative and quantitative research within mixed method research designs: a methodological review. International journal of nursing studies, v. 48, n. 3, p. 369-383, 2011.

POLIT, Denise F.; BECK, Cheryl Tatano. Generalization in quantitative and qualitative research: Myths and strategies. International journal of nursing studies, v. 47, n. 11, p. 1451-1458, 2010.

$\mathrm{RICH}$, Michael; GINSBURG, Kenneth R. The reason and rhyme of qualitative research: why, when, and how to use qualitative methods in the study of adolescent health. Journal of Adolescent health, v. 25, n. 6, p. 371-378, 1999.

RINGLE, Christian M.; DA SILVA, Dirceu; BIDO, Diógenes de Souza. Modelagem de equações estruturais com utilização do SmartPLS. REMark, v. 13, n. 2, p. 54, 2014.

SANDELOWSKI, Margarete. Combining qualitative and quantitative sampling, data collection, and analysis techniques in mixed-method studies. Research in nursing \& health, v. 23, n. 3, p. 246-255, 2000. 
SAUERBRONN, João Felipe Rammelt; AYROSA, Eduardo André Teixeira. Concerning convergence and the methodological practice of interpretive interactionism in academic marketing research. Revista de Administração Contemporânea, v. 14, n. 5, p. 854-870, 2010.

SILVERMAN, David. Interpretação de dados qualitativos: métodos para análise de entrevistas, textos e interações. São Paulo: Bookman, 2009.

SWEENEY, Dennis J.; WILLIAMS, Thomas A.; ANDERSON, David R. Estatística aplicada à administração e economia. São Paulo: CENGAGE Learning, 2013.

STARK, Oded; JAKUBEK, Marcin; KOBUS, Martyna. A bitter choice turned sweet: How acknowledging individuals' concern at having a low relative income serves to align utilitarianism and egalitarianism. Journal of Evolutionary Economics, v. 25, n. 3, p. 541-557, 2015.

TEIXEIRA, Juliana Cristina; NASCIMENTO, Marco César Ribeiro; CARRIERI, Alexandre de Pádua. Triangulação entre métodos na administração: gerando conversações paradigmáticas ou meras validações" convergentes"? Revista de Administração Pública-RAP, v. 46, n. 1, 2012.

TROTTER, Robert T. Qualitative research sample design and sample size: Resolving and unresolved issues and inferential imperatives. Preventive medicine, v. 55, n. 5, p. 398-400, 2012.

VERGARA, Sylvia Constant; CALDAS, Miguel Pinto. Paradigma interpretacionista: a busca da superação do objetivismo funcionalista nos anos 1980 e 1990. RAE-revista de administração de empresas, v. 45, n. 4, p. 66-72, 2005.

WESTERMAN, Michael A. What counts as "good" quantitative research and what can we say about when to use quantitative and/or qualitative methods? New ideas in psychology, v. 24, n. 3, p. 263-274, 2006.

WESTERMAN, Michael A. Conversation analysis and interpretive quantitative research on psychotherapy process and problematic interpersonal behavior. Theory \& Psychology, v. 21, n. 2, p. 155-178, 2011.

YANCHAR, Stephen C. Beyond the qualitative-quantitative divide: How to proceed? New Ideas in Psychology, v. 24, n. 3, p. 275-281, 2006.

YIN, Robert K. Estudo de Caso: Planejamento e Métodos. São Paulo: Bookman, 2008. 